\title{
Subchannel Analysis, CFD Modeling and Verifications, CHF Experiments and Benchmarking
}

\author{
Baowen Yang, ${ }^{1}$ Yassin A. Hassan, ${ }^{2}$ Jianqiang Shan, ${ }^{1}$ Bin Zhang, \\ Junli Gou, ${ }^{1}$ and Liangzhi Cao ${ }^{1}$ \\ ${ }^{1}$ School of Nuclear Science and Technology, Xian Jiaotong University, No. 28 Xianning West Road, Xian 710049, China \\ 2 335Q Zachry Engineering Center, Department of Nuclear Engineering, Texas A\&M University, College Station, TX 77843-3133, USA
}

Correspondence should be addressed to Baowen Yang; bwy@mail.xjtu.edu.cn

Received 29 June 2014; Accepted 29 June 2014; Published 20 July 2014

Copyright (C) 2014 Baowen Yang et al. This is an open access article distributed under the Creative Commons Attribution License, which permits unrestricted use, distribution, and reproduction in any medium, provided the original work is properly cited.

In the heart of light water reactors, though the nuclear fuel elements are a source of energy, they are also the root of potential threat for nuclear accidents. The investigation and understanding of the multiphase, multiphysics, and multiscale subchannel systems in the reactor core are the key to an economical and safe operation of a nuclear power plant. There are several unique features associated with such subchannel systems including strong interchannel interaction, flow structure coupling, nonuniform heat source (both axially and radially), periodic flow obstruction and mixing through mixing vane grids along the length of fuel assembly, and strong multiphysics coupling. These unique features present the upmost challenging and critical task for nuclear fuel efficiency and safety. As one of the most active areas of nuclear thermal-hydraulics, research on subchannel analysis and CFD modeling and verifications, as well as rod bundle CHF experiments and benchmarking is of great interest to the nuclear fuel design and reactor safety. Due to the technical difficulty, time consumption, and high cost involved in the conventional large scale subchannel mixing and rod bundle CHF experiments for light water reactors, CFD modeling has become the major trend in predicting the local conditions in complicated subchannel geometry. The advancements in CFD modeling from single phase turbulent mixing to multiphase CFD modeling techniques involving Euler-Euler two-fluid modeling, large Eddy simulation (LES), direct numerical simulation (DNS), or Lattice Boltzmann methods (LBM), and so forth, helped reveal ample details of local conditions in the complicated subchannel system.
It is with great anticipation that the advancement of CFD modeling might facilitate further understanding of various thermal-hydraulic phenomena, such as excursion of flow instability and turbulent mixing, as well as various mechanisms leading to the limiting conditions of critical heat flux (CHF). However, the progress in modeling efforts have continued facing monumental challenges in single and multiphase experiments involving interphase tracking, nonintrusive measurement in highly obstructed narrow channels and flow visualization concerning wide ranges of thermalhydraulic conditions (from low pressure, low temperature to high pressure, high temperature), as well as validation, verification, and uncertainty qualification of rod bundle experimental data for CFD benchmarking. This special issue invited investigators to contribute original research articles as well as review papers across the following topics: (1) recent development in fuel design tools, CFD modeling, benchmarking, and verification techniques; (2) VV/UQ and rod bundle CHF experiments and verifications; (3) mechanistic modeling and empirical DNB correlations for BWR and PWR fuel; (4) effect of parameters on CHF; (5) reactor core protection against DNB; (6) fuel reliability and inspection.

Six papers were selected for this special issue. The paper by B. Yang et al. titled "Uniform versus nonuniform axial power distribution in rod bundle CHF experiments" presents potential limitations of using uniform rod bundle CHF data for CHF correlation development of light water reactors with nonuniform axial power distribution (APD). The paper "Effect of flow blockage on the coolability during reflood in 
a $2 \times 2$ rod bundle" by K. Kim et al. reveals the experimental study on the thermal behavior and effect of the ballooned region on the coolability using a $2 \times 2$ rod bundle test facility during the reflood phase of a large-break-loss-ofcoolant accident (LBLOCA) in a pressurized-water reactor (PWR). The paper of J. Shan et al. titled "Subchannel anal$y$ sis of wire wrapped SCWR assembly" provides subchannel analysis and experimental investigation of a wire-wrapped SCWR assembly. In the paper "Direct numerical simulation and visualization of subcooled pool boiling," by T. Kunugi and Y. Ose, perform direct numerical simulation of the subcooled pool boiling phenomena in order to clarify the heat transfer characteristics and discuss the mechanism in their paper. The influence of outer strap design on fuel assembly thermal hydraulic performance is studied using a commercial computational fluid dynamics (CFD) code, ANSYS CFX, and a subchannel analysis code, FLICA, in the paper entitled "Influence of spacer grid outer strap on fuel assembly thermal hydraulic performance" by J. Yan et al. Finally, the paper of C. Peña-Monferrer et al. titled "CFD turbulence study of PWR spacer-grids in a rod bundle" analyzes the turbulence effects of different types (a split-type and a swirl-type) of spacergrid geometries on single-phase turbulent mixing in a PWR (pressurized water reactor) rod bundle.

\title{
Acknowledgment
}

The editors of this special issue express their sincere gratitude to all authors of all the remitted papers (selected or not) for their contribution.

\author{
Baowen Yang \\ Yassin A. Hassan \\ Jianqiang Shan \\ Bin Zhang \\ Junli Gou \\ Liangzhi Cao
}




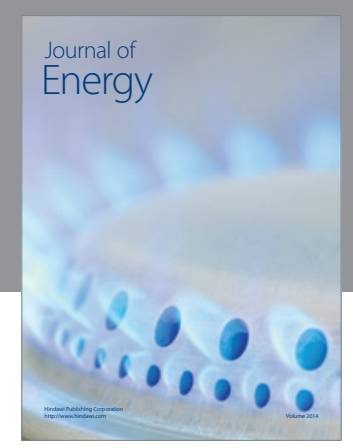

Journal of

Industrial Engineering
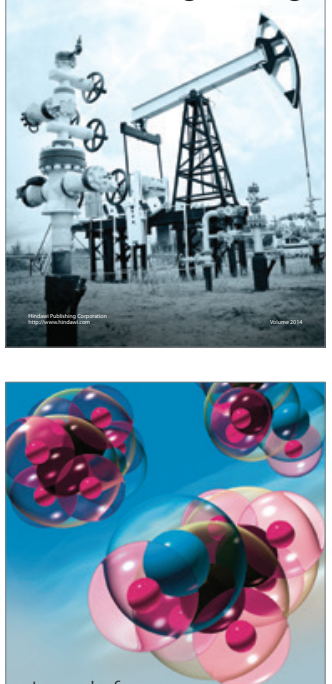

Fuels
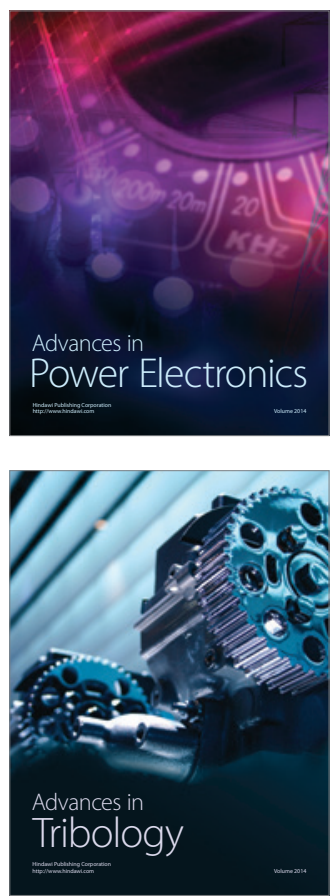

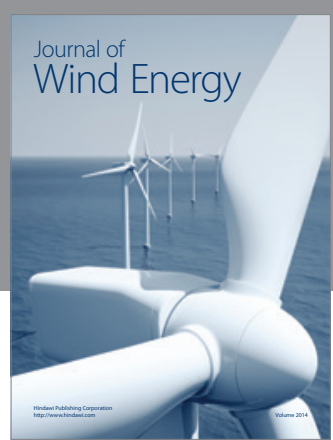

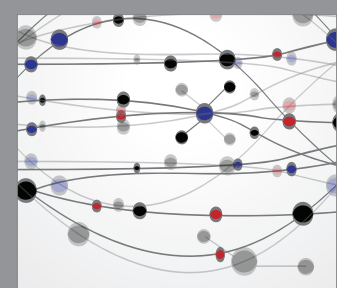

The Scientific World Journal

Submit your manuscripts at http://www.hindawi.com

Journal of

Structures
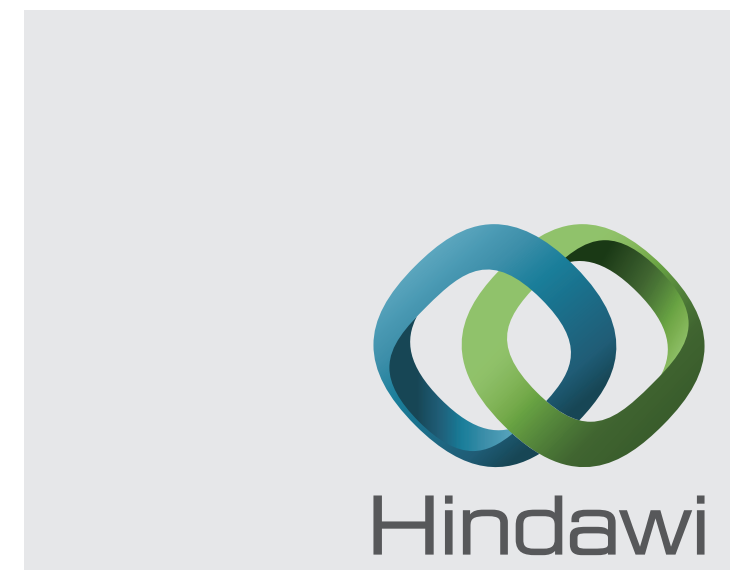

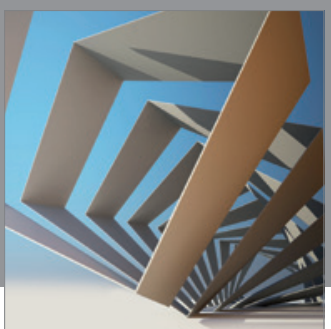

Rotating

Machinery
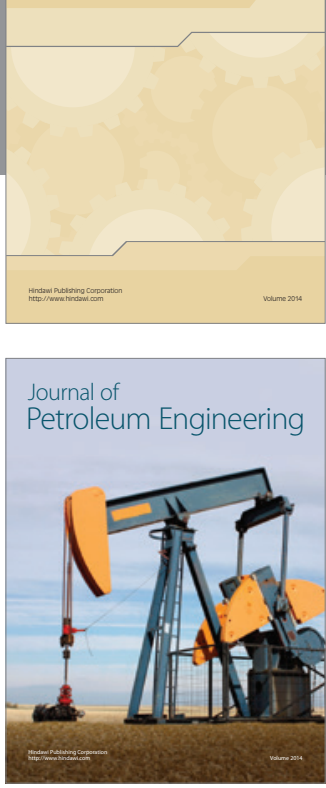

Journal of

Solar Energy
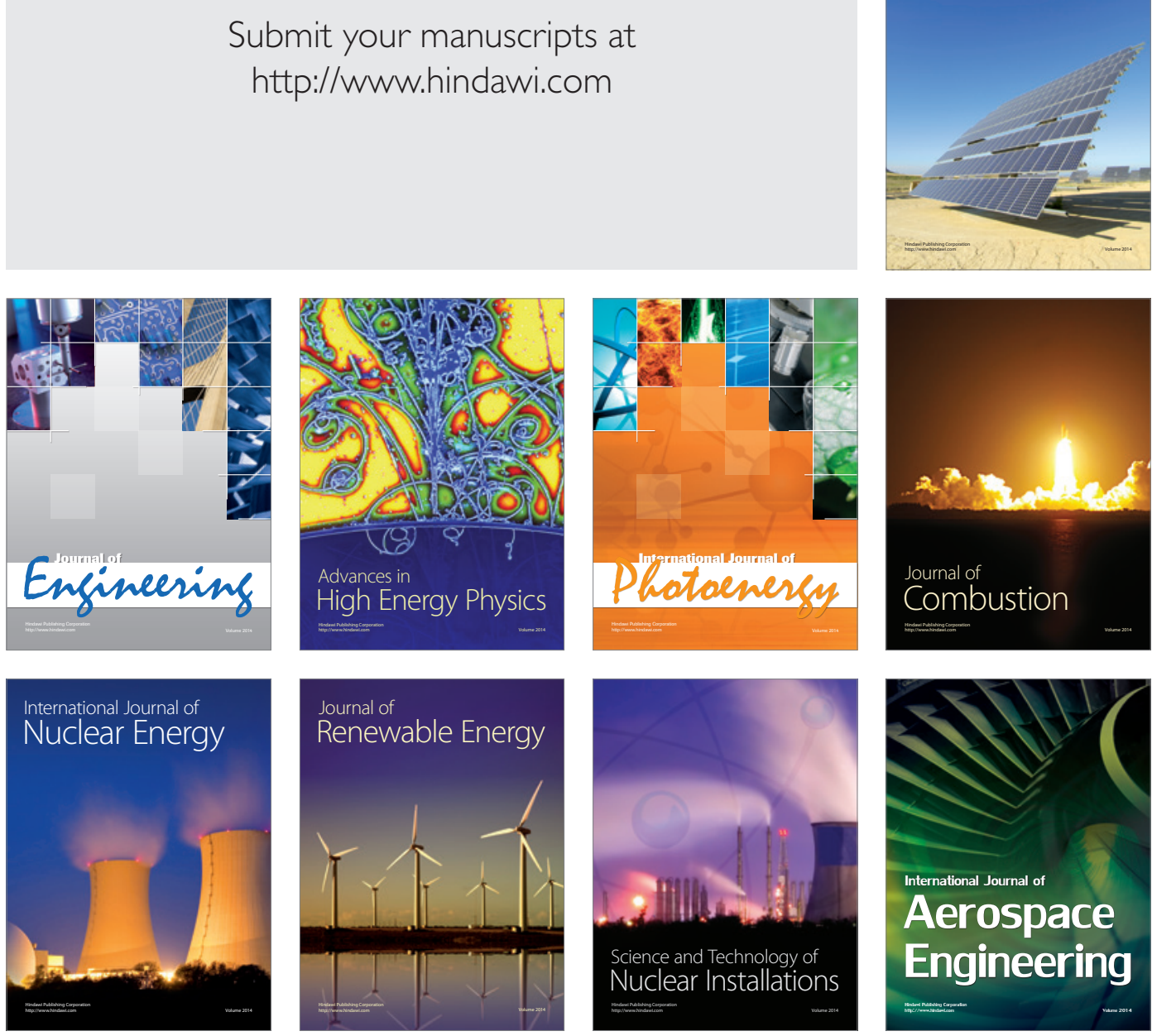